Proceedings of the International Conference on Oxide Materials for Electronic Engineering, May 29-June 2, 2017, Lviv

\title{
Utilization of Electromagnetic Tomography for Ferrite Rings Testing
}

\author{
P. NowaK ${ }^{a, *}$, M. Nowicki ${ }^{b}$, K. Gromada ${ }^{c}$ And R. SzeWCZYK ${ }^{b}$ \\ ${ }^{a}$ Industrial Research Institute for Automation and Measurements PIAP, \\ Al. Jerozolimskie 202, 02-486 Warsaw, Poland \\ ${ }^{b}$ Institute of Metrology and Biomedical Engineering, Warsaw University of Technology, \\ św. A. Boboli 8, 02-525 Warsaw, Poland \\ ${ }^{c}$ Institute of Automatic Control and Robotics, Warsaw University of Technology, \\ św. A. Boboli 8, 02-525 Warsaw, Poland
}

\begin{abstract}
Paper presents utilized innovative setup for eddy current tomography and possibility of its utilization in testing oxide materials such as ferrites. Previously reported tests concerned materials with high conductivity which is the most typical usage of eddy current tests. Described tomography setup is designed for testing axisymmetric objects thus typical ferrite ring was selected for exemplary testing. Tests were conducted on ring in original state. Afterwards reference defect was created on element and measurements were repeated. Significant difference between tests results were observed, thus potential for utilization in controlling of ferrite rings manufacturing process was confirmed. Finite element method simulations were applied in order to confirm the measurement results. Calculations were conducted in open-source finite element method software, which solves the Maxwell equations in the $\mathrm{A}-\mathrm{V}$ form. Modelling results confirm possibility of finite element method-based inverse tomography transformation
\end{abstract}

DOI: 10.12693/APhysPolA.133.1045

PACS/topics: 85.80.Jm, 87.10.Kn

\section{Introduction}

Tomography tests are one of the most intensively developed form of non-destructive testing of many mechanical elements. The most important advantage of tomography is the unique possibility of obtaining data about both shape and the nature of discontinuities in the tested element. Typically used X-ray tomography is a source of many risks typical for ionizing radiation techniques. This results in significant cost and difficulties for usage in industrial environment, and may sometimes be dangerous for operators.

Eddy current tomography is a suitable alternative [1]. It allows for parallel measurements of resistivity and magnetic susceptibility of material in the element tested [2]. This results in new possibilities for detection of discontinuities in structures while performing tests in industrial conditions. Previously reported research $[1,3]$ were conducted on conductive materials with different magnetic permeability. This paper focuses on testing the low-conductive material with high magnetic permeability. This significantly limits the induction of eddy currents. On the other hand, high permeability objects influence the distribution of magnetic field, which influences the measured signals, more as in AC magnetic induction tomography $[4,5]$.

* corresponding author; e-mail: pnowak@piap.pl

\section{Tomography measurements}

Tests were conducted on an electromagnetic tomography setup [1]. Tested element was moved linearly between the driving coil and the measuring coil. Additionally, in each linear position object is rotated. $2 \mathrm{kHz}$ sine current from the generator powers the 100-turns driving coil, which induces sine magnetic field. Depending on tested element material's conductivity and permeability, altered signal is sensed by 100-turns measuring coil. After proper signal conditioning, data about amplitude and phase shift (respectively to the driving coil signal) are measured.

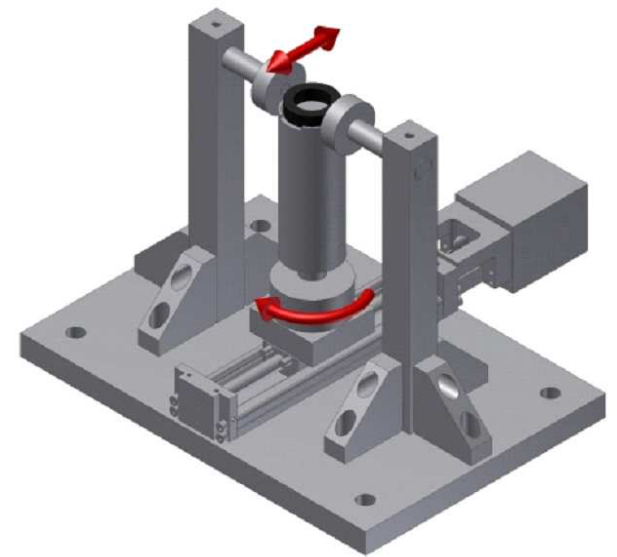

Fig. 1. 3D model of utilized test stand with arrows marking the movement of tested object. 

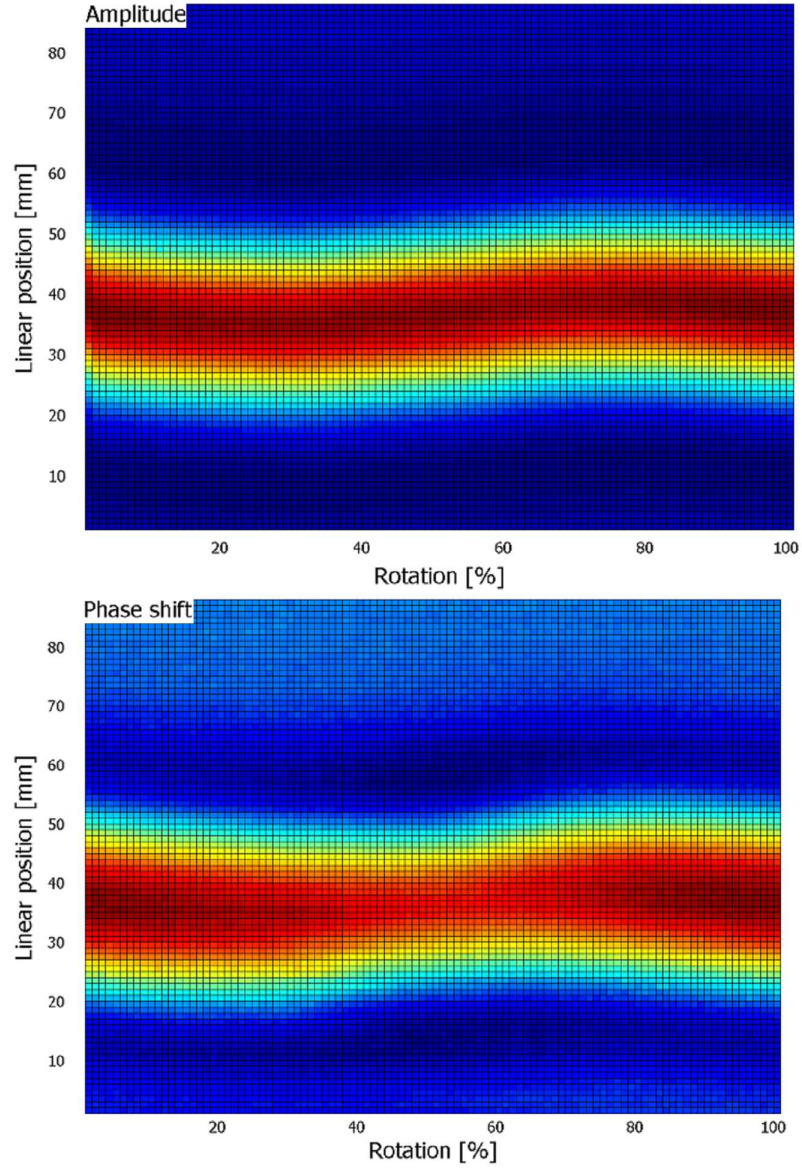

Fig. 2. Obtained measurement results for object in delivery condition.

The measurement setup was designed for testing axisymmetric objects, so a typical ferrite ring was selected for tests. 3D model of utilized test stand, with the tested object, is presented in Fig. 1.

Ring was measured on the setup firstly in the delivery condition and secondly after making a reference defect (longitudinal cut on the edge of the ring). Obtained results are presented in Fig. 2 and Fig. 3.

In the top graph an amplitude measurement is presented, whereas bottom graph shows value of the phaseshift. Each $X Y$ point on the graph corresponds to single measurement position and the colour of the point ( $Z$ axis projected onto the graph plane) represents the linearly normalized value of the measured parameters (signal in corresponding measurement points). Results presented in Fig. 2 (for object in delivery condition with no defects) present significant change in the rotation function. This is caused by eccentricity of the object placement in the measurement setup. With the knowledge about the state of the object (lack of defects) the eccentricity may be compensated numerically.

Algorithm for eccentricity compensation bases on lowpass far infrared (FIR) interpolation. This way maximum of each column (rotational position) is localised and vec-
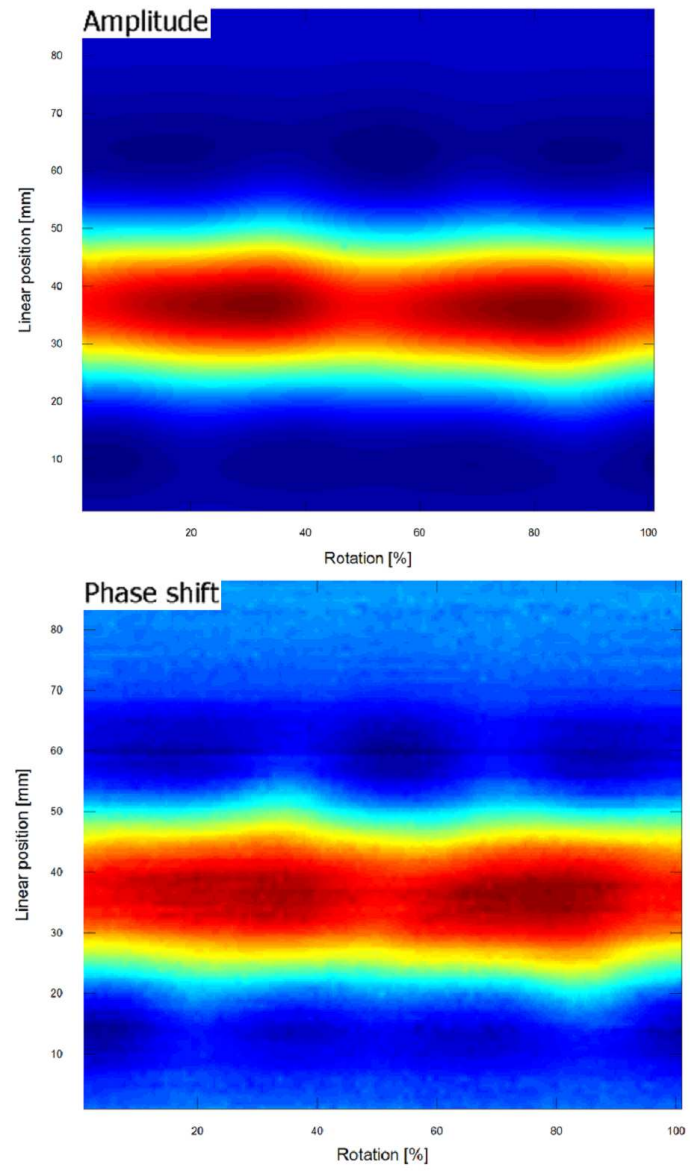

Fig. 3. Obtained measurement results for object with reference defect.

tor of eccentricity is determined. Aberration is limited by applying corrective translation, which can be fractal of single cell, improving its reliability. Results of eccentricity compensation are presented in Fig. 4 and confirm proper implementation of algorithm. After the compensation of eccentricity, a differential analysis of the results was conducted. Results presented in Fig. 5 present defect's influence on the obtained measurement results.

\section{FEM modelling results}

In order to confirm influence of the reference defect on the measurement result (mostly, the influence of high permeability material on the magnetic field distribution) and to present possibility of inverse tomography transformation, a FEM modeling of the simplified problem was conducted.

Simulations were made in the open-source ElmerFEM software, which utilizes first order Whitney elements for solving the Maxwell equations in frequency domain [6]. Usage of the Whitney edge elements [7] makes solver suitable only to $3 \mathrm{D}$ problems.

Tomography setup was simplified to the two-coil setup with tested element between them. Exciting coil was modeled as a single turn coil, whereas measurement coil was simplified as a constant disk for better representation 

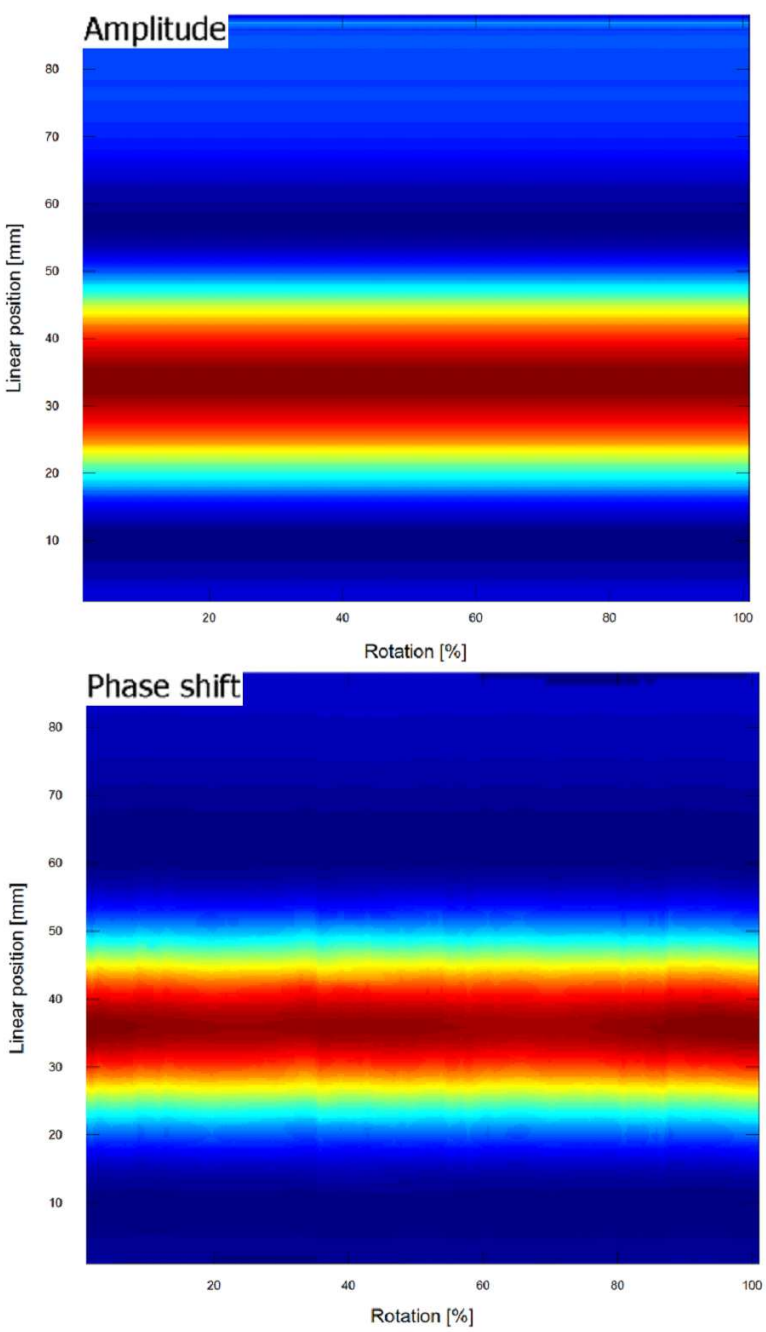

Fig. 4. Measurement results for object in delivery condition after numerical eccentricity compensation.

of integration of magnetic field phenomena. Whole setup was placed in a large modeling sphere, which serves two purposes during modelling. Firstly, sphere provides place for modelling of distribution of magnetic field in the air around the objects [8]. Secondly, external surface of the sphere is utilized for applying proper Dirichlet boundary condition for the differential equation solvers.

Simulations were conducted for three object's models - one in delivery condition, and two with reference defect. During simulations exciting coil was powered with alternate current, whereas the magnetic potential value on the external surface of the modelling sphere was forced 0 (the Dirichlet condition). Obtained distributions of magnetic field in objects are presented in Figs. 6-8. Disturbance of magnetic field distribution, caused by the element defect, is clearly visible. Based on the forward modeling, inverse tomography transformation may be developed. Due to proper reconstruction of tested element shape, based on the tomography measurements, failure in production process can be detected.
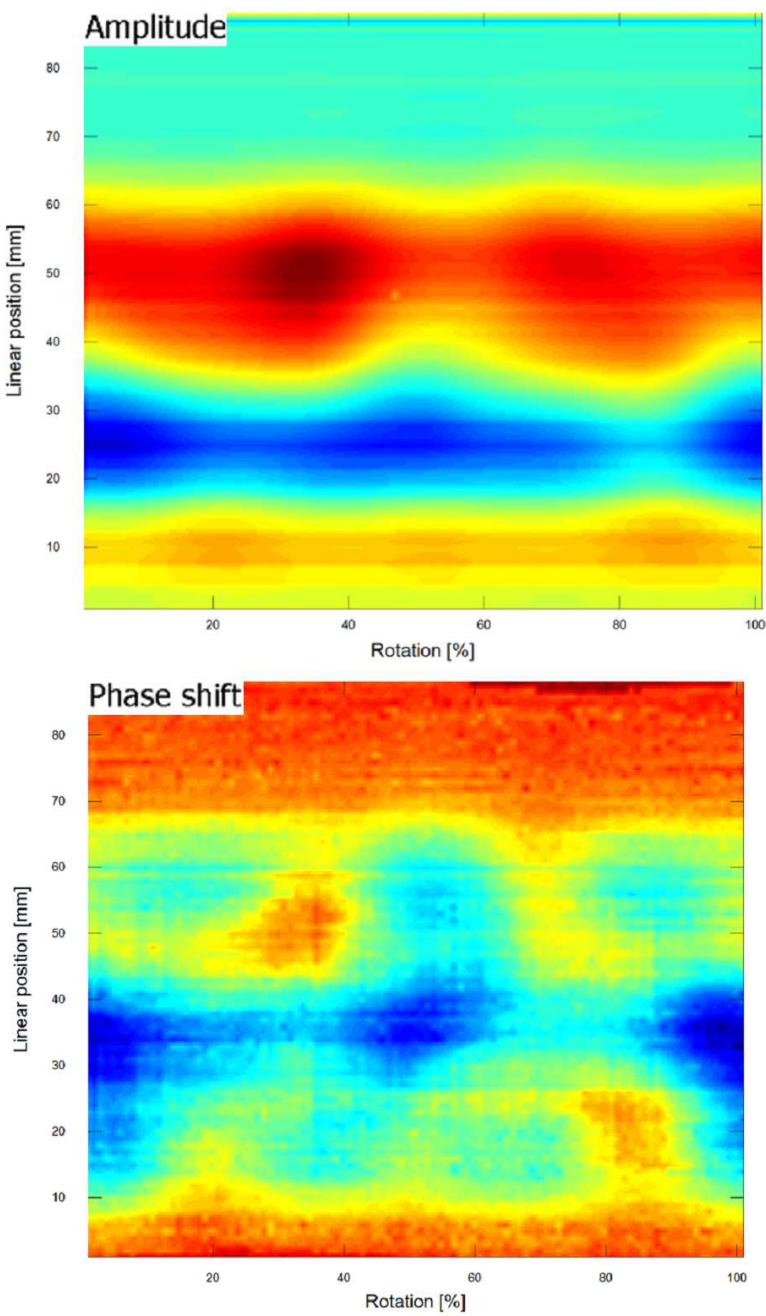

Fig. 5. Results of differential analysis of measurements results.

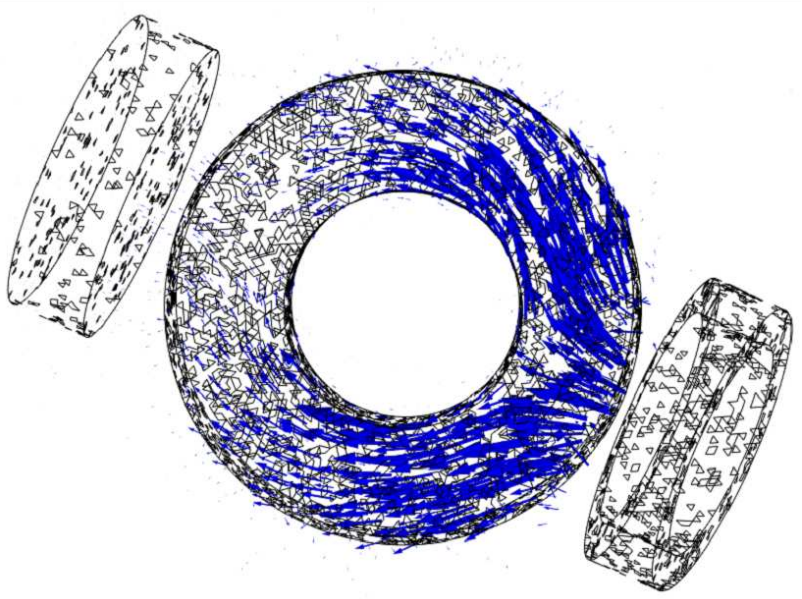

Fig. 6. Distribution of magnetic field vectors in object without defects. 


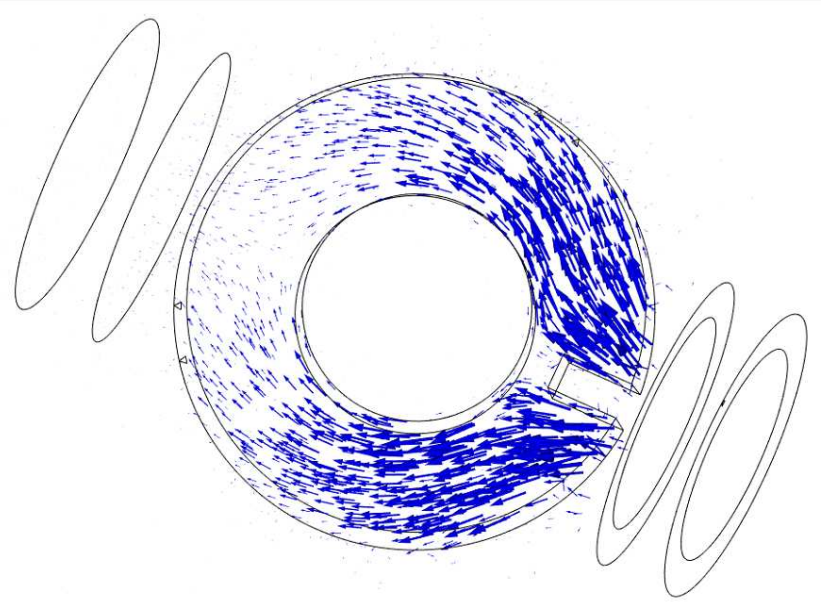

Fig. 7. Distribution of magnetic field vectors in object with defect parallel to the coils axis.

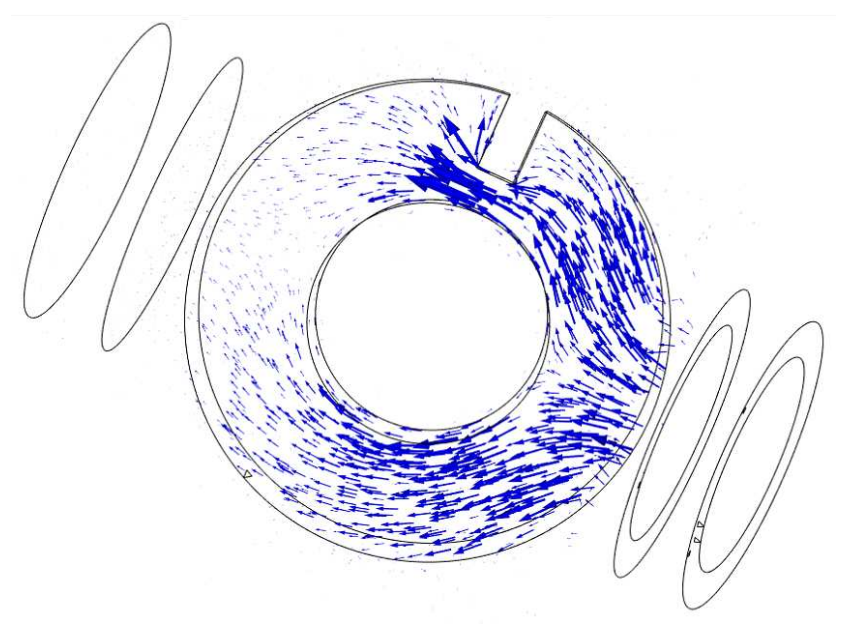

Fig. 8. Distribution of magnetic field vectors in object with defect perpendicular to the coils axis.

\section{Conclusions}

Innovative method for testing commonly produced element was developed as presented. Measurements were conducted on typical ferrite ring with and without reference defect. Differential analysis of the measurement results confirms influence of the object structure on the obtained results, which was also confirmed with FEM analysis.
Further research shall concern development of inverse tomography transformation, which will lead to proper and reliable reconstruction of the shape of elements. Other proposition is FEM-based development of reference results database for different types of objects and their defects.

\section{Acknowledgments}

This work was partially supported by the statutory founds of Institute of Metrology and Biomedical Engineering, Warsaw University of Technology (Poland).

\section{References}

[1] J. Salach, in: Advances in Intelligent Systems and Computing, Vol. 317, Eds. J. Awrejcewicz, R. Szewczyk, M. Trojnacki, M. Kaliczyńska, Springer, Berlin 2015, p. 373.

[2] D. Premel, A. Mohammad-Djafari, IEEE Trans. Magn. 31, 2000 (1995).

[3] P. Nowak, M. Nowicki, A. Juś, R. Szewczyk, Acta Phys. Pol. A 131, 1168 (2017).

[4] M. Soleimani, W.R.B. Lionheart, IEEE Trans. Med. Imaging 25, 1521 (2006).

[5] M. Soleimani, W. Lionheart, A. Peyton, X. Ma, in: Proc. 3rd World Congress on Industrial Process Tomography, Banff (Canada), 2003, p. 252.

[6] R. Szewczyk, J. Salach, J. Ruokolainen, P. Råback, K. Stefko, M. Nowicki, in: Advances in Intelligent Systems and Computing, Vol. 352, Eds. R. Szewczyk, C. Zieliński, M. Kaliczyńska, Springer, Berlin 2015, p. 219.

[7] A. Bossavit, IEE Proc. A - Phys. Sci. Measur. Instrum. Manag. Educ. 135, 493 (1988).

[8] P. Nowak, R. Szewczyk, in: Advances in Intelligent Systems and Computing, Vol. 543, Eds. R. Szewczyk, M. Kaliczyńska, Springer, Berlin 2017, p. 75. 